Review

\title{
A Review of the Biochemistry, Metabolism and Clinical Benefits of Thiamin(e) and Its Derivatives
}

\section{Derrick Lonsdale}

Preventive Medicine Group, Derrick Lonsdale, 24700 Center Ridge Road, Westlake, OH 44145, USA

\begin{abstract}
Thiamin(e), also known as vitamin B1, is now known to play a fundamental role in energy metabolism. Its discovery followed from the original early research on the 'anti-beriberi factor' found in rice polishings. After its synthesis in 1936, it led to many years of research to find its action in treating beriberi, a lethal scourge known for thousands of years, particularly in cultures dependent on rice as a staple. This paper refers to the previously described symptomatology of beriberi, emphasizing that it differs from that in pure, experimentally induced thiamine deficiency in human subjects. Emphasis is placed on some of the more unusual manifestations of thiamine deficiency and its potential role in modern nutrition. Its biochemistry and pathophysiology are discussed and some of the less common conditions associated with thiamine deficiency are reviewed. An understanding of the role of thiamine in modern nutrition is crucial in the rapidly advancing knowledge applicable to Complementary Alternative Medicine. References are given that provide insight into the use of this vitamin in clinical conditions that are not usually associated with nutritional deficiency. The role of allithiamine and its synthetic derivatives is discussed. Thiamine plays a vital role in metabolism of glucose. Thus, emphasis is placed on the fact that ingestion of excessive simple carbohydrates automatically increases the need for this vitamin. This is referred to as high calorie malnutrition.
\end{abstract}

Keywords: disulfide - oxidative stress - thiamine deficiency

\section{Introduction}

Nutritional deficiency is widespread throughout the world. Most of this is due to economic disadvantage and overcrowding. In the developing world bad cooking habits may also account for this problem. Although this form of malnutrition certainly exists in the Unites States, a common form is associated with moderate affluence and is related to ingestion of simple carbohydrate in all its different forms. This is referred to in the text as high calorie malnutrition. Marginal deficiency of this nature results in typical symptoms that are sometimes referred to by physicians as functional (1). Because thiamine is a major factor in the metabolism of glucose, it has long been known that ingestion of simple carbohydrates,

For reprints and all correspondence: Derrick Lonsdale, Preventive Medicine Group, Derrick Lonsdale, 24700 Center Ridge Road, Westlake, OH 44145, USA. Tel: +1-440-835-0104; Fax: +1-440-871-1404; E-mail: dlonsdale@pol.net processed in the body mainly to glucose, automatically increases the need for dietary thiamine. Thus, high calorie malnutrition is commonly associated with relative thiamine deficiency, irrespective of its fortification in food substances. This review will discuss the biochemistry of thiamine in animal systems and the various clinical expressions of its dietary deficiency and dependency. It will then describe the clinical uses of the vitamin and its many derivatives that have resulted in beneficial responses in a variety of diseases in man and animals. Its place in nutrient-based Complementary Alternative Medicine is emphasized.

\section{Historical Facts}

In 1965, a Vitamin B Research Committee in Japan provided detailed information on the history of the scourge of beriberi, now known to be associated with deficiency of thiamine (2). The disease has been known since antiquity and its original

(C) The Author (2006). Published by Oxford University Press. All rights reserved.

The online version of this article has been published under an open access model. Users are entitled to use, reproduce, disseminate, or display the open access version of this article for non-commercial purposes provided that: the original authorship is properly and fully attributed; the Journal and Oxford University Press are attributed as the original place of publication with the correct citation details given; if an article is subsequently reproduced or disseminated not in its entirety but only in part or as a derivative work this must be clearly indicated. For commercial re-use, please contact journals.permissions@oxfordjournals.org 
name of 'Kakke' can be found in documents as early as 808 . The mortality per 100 of the Japanese population in the 20th century began to decline from 30 in the 1920s to 0.5 in 1969 after its thiamine deficient etiology was discovered.

In 1965, the morbidity from beriberi was not accurately known. A study of 375 patients seen in Tokyo Medical and Dental College Hospital revealed that 109 (29\%) had at least two of the major beriberi signs of hyporeflexia, hypesthesia, edema, lowered diastolic pressure and tenderness by grasping the calf muscles.

A curious phenomenon observed was that death from the disease had a regular seasonal variation, with the peak in August and September, found every year between 1899 and 1938 when records were kept. There was also increased morbidity in working areas where humidity was high. The incidence of infantile beriberi followed the same pattern, and was higher in males than in females by a ratio of $1.76: 1.0$.

That beriberi was closely related to food was first pointed out by Takaki, a Japanese navy surgeon. In 1882, a naval vessel from Japan returned after 272 days with $61 \%$ of its crew affected with beriberi. In 1884, Takaki loaded another warship with dry milk and meat. After a voyage of 287 days, only 14 of the crew had succumbed to the disease. Takaki concluded that the disease was caused by a lack of nitrogenous food in association with excessive intake of non-nitrogenous food.

In 1890, Eijkman accidentally found that fowls, when fed polished rice, died soon from paralysis and that the peripheral nerves revealed histological changes analogous to those seen in human beriberi. In 1901, Grujns found that starch feeding had the capacity to produce the same pattern in fowls.

It gradually became evident that polished rice ingestion caused beriberi and that rice bran germ, barley and red beans, when taken together with polished rice, would prevent the disease. Funk and Cooper eventually isolated a substance from rice polishing and called it 'Vitamine'. This early research eventually led to the discovery of the active principle and it was named thiamine.

Beriberi was the first disorder in which the term 'deficiency disease' was used. Polyneuritis in fowls restricted to a diet of polished rice appeared to be similar to the polyneuritis in beriberi victims. This prompted Eijkman in the Dutch East Indies to study the problem. He showed that the polyneuritis in fowls could be prevented by diet and reported the results of his studies in 1896-97. The 'anti-beriberi' substance was crystallized from rice polishings by Jansen and Donath in 1926 (3). The structure and synthesis of thiamine was reported by Williams (4), and the ground-breaking work on its biochemical action was studied by Peters (5).

\section{Biochemistry}

\section{Thiamine}

In various publications, it is spelled with and without the ' $\mathrm{e}$ '. The ' $\mathrm{e}$ ' was dropped when it was found that it was not an amine. The word is used here with an ' $\mathrm{e}$ ' is used here since it appears still to be more commonly used. It consists of a pyrimidine ring (2,5-dimethyl-6-aminopyrimidine) and a thiazolium ring (4-methyl-5-hydroxy ethyl thiazole) joined by a methylene bridge.

\section{Natural Sources}

Thiamine is present in lean pork and other meats, wheat germ, liver and other organ meats, poultry, eggs, fish, beans and peas, nuts, and whole grains. Dairy products, fruit and vegetables are not good sources. The RDA is $0.5 \mathrm{mg}$ per $1000 \mathrm{kcal}$, adequate for a healthy individual consuming a healthy diet. Considerable losses occur during cooking or other heat-processing of food. Polyphenolic compounds in coffee and tea can inactivate thiamine so that heavy use of these beverages could compromise thiamine nutrition (6).

\section{Thiamine monophosphate}

A review of the thiamine literature revealed that little or nothing is known about whether there is a specific biochemical role of thiamine monophosphate (TMP) other than it being a step from free thiamine to the intracellular synthesis of thiamine diphosphate and triphosphate. It may be analogous to the stepwise phosphorylation of adenosine.

\section{Thiamine pyrophosphate (diphosphate)}

Although the basic chemistry of thiamine is now fairly well known, there is a great deal of information still missing. It can be stated that it is an incredibly active molecule and plays a part in many different aspects of energy metabolism.

Not long after thiamine was recognized as a nutritional requirement for many forms of life, it was found that its pyrophosphate ester, thiamine diphosphate (TPP) was a cofactor for enzymatic reactions that cleave alpha-keto acids. It is now well known that TPP activates decarboxylation of pyruvate in the pyruvate dehydrogenase complex. This complex is a group of enzymes and cofactors that form acetyl CoA that condenses with oxaloacetate to form citrate, the first component of the citric acid cycle. Since pyruvate is derived from glucose via the Embden-Meyerhof pathway, it should be emphasized that the energy drive from oxidation of glucose is highly dependent upon TPP. It is also a cofactor in the decarboxylating component of alpha-ketoglutarate dehydrogenase, an important link in the citric acid cycle.

A third enzyme complex that requires TPP as a cofactor is the macromolecular aggregation that decarboxylates the keto acids derived from leucine, isoleucine and valine, the branched chain amino acids. The fourth important enzyme requiring TPP is transketolase, an enzyme that occurs twice in the pentose shunt. The functions of this pathway are to provide pentose phosphate for nucleotide synthesis and to supply reduced NADP for various synthetic pathways. Activity of this enzyme has been used for many years as the best functional test for evidence of thiamine deficiency (7). Since the pentose shunt occurs in erythrocytes, these cells become 
convenient for performing the study as a routine method of detecting deficiency.

\section{Thiamine Triphosphate}

As early as 1938, Minz (8) first suggested a relationship between thiamine and nervous excitation when he observed that thiamine was released into the bathing medium when the pneumogastric nerve, taken from an ox, was stimulated. Cooper and Pincus (9) reviewed the evidence that there was a possibility that thiamine has a neurophysiological function that is distinct from its activity as a coenzyme. They reported that thiamine appears to be uniformly distributed throughout the nervous system and appears to be highly localized in membrane structures. More recently, the distribution of thiamine was studied in rats (10). After intracerebroventricular injection of labeled thiamine the distribution of its radioactive esters was found to be as follows: thiamine, 8-12\%; TMP, 12-14\%; TPP, 72-74\%; and thiamine triphosphate (TTP), $2-3 \%$.

Cooper and Pincus (9) had reported that they, and other investigators, had confirmed that nerve stimulation in experimental animal systems resulted in decline of the level of TPP and TTP in the preparation. The released compounds were in the form of TMP and free thiamine. This phenomenon made it difficult to interpret the function of the vitamin in nerve conduction. Bettendorff et al. (11) discussed the physiological significance of TTP in the main electric organ of Electrophous electricus that is particularly rich in TTP, representing $87 \%$ of the total thiamine content of this tissue. The real substrate of TTP phosphatase, they said, is probably a $1: 1$ complex of $\mathrm{Mg}^{2+}$ and TTP. Incubation of rat brain homogenates with thiamine and TPP leads to synthesis of TTP (12) and further study suggested that TTP is an activator of chloride channels having a large unit conductance (13). In mammalian tissues TTP concentrations are regulated by a specific thiamine triphosphatase (14). The role of TTP is, however, incompletely known at the present time.

\section{Thiamine Transporter}

The SLC gene family of solute carriers is a family of three transporter proteins with significant structural similarity, transporting substrates with different structure and ionic charge. SLC19A1 mediates the transport of reduced folate and its analogs and SLC19A2 mediates the transport of thiamine. SLC19A3 is also capable of transporting thiamine $(15,16)$.

\section{Thiamine Deficiency Disease}

The classical syndrome caused primarily by thiamine deficiency in humans is beriberi, in which the benefit of thiamine in prevention and treatment is uncontested $(17,18)$. In older texts it has been divided into categories known as 'wet', 'dry', 'childhood', 'infantile' and Wernicke-Korsakoff syndrome. More modern knowledge recognizes that the symptoms and signs may or may not be associated with edema and vary according to the age of the patient. Its presentation also depends on the association with other vitamin deficiencies and the ingestion of empty calories, particularly in the form of simple carbohydrate. Description of symptoms and signs associated with the various aspects of the disease have been thoroughly reviewed many years ago (19) and need not be reiterated in this review. Some of the lesser known and uncommon manifestations of thiamine deficiency are discussed later in the text. It is to be emphasized that they are based on only a few reported case studies and that there are insufficient data in the literature to support these associations conclusively.

Although the vitamin has been known since the 1930s and experimental work has been performed in both animals and man, the overall mechanisms resulting from its deficiency are still not completely understood. Platt (20), one of the earliest investigators of beriberi stated that a conference in Princeton had stressed that thiamine deficiency (TD) and beriberi were not synonymous terms, perhaps emphasizing the importance of the inter-relations of other nutrients with thiamine. The clinical features of beriberi have long been largely forgotten in modern medicine since it has been assumed that the classic deficiency diseases do not occur in developed societies. It is, however, important to state that dysfunction in the autonomic system was a prominent part of the clinical expression of beriberi (21) and reversible autonomic dysfunction, associated with evidence of high calorie malnutrition, has been reported in recent times in the United States $(22,23)$. Sweating, tachycardia, dermographia, wide unstable pulse pressure, attention deficit and other symptoms of autonomic dysfunction in children have been reported as functional dysautonomia (24).

Limited data are available on the relation between thiamine requirements and the intake of carbohydrates. A study has been reported that investigated the influence of stepwise increases of carbohydrate intake on the status of thiamine in healthy volunteers under isocaloric conditions (25). An increase of dietary carbohydrate intake caused a decrease of plasma and urine levels of thiamine without affecting enzyme activities.

Glyoxals are reactive alpha-oxoaldehydes that are formed endogenously from sugars, the levels of which are increased in various pathological conditions associated with hyperglycemia and thiamine deficiency. A study found that low concentrations of glyoxal markedly increased the susceptibility of hepatocyte glutathione (GSH) to oxidation by hydrogen peroxide. It increased cytotoxicity by compromising the cellular antioxidant enzyme system. At higher concentrations it was cytotoxic towards hepatocytes, attributed to GSH depletion, oxidative stress and mitochondrial toxicity. Glyoxal toxicity was prevented by increasing its metabolic breakdown with thiamine or $\mathrm{NADP}(\mathrm{P}) \mathrm{H}$ generators. Toxicity was increased in GSH or thiamine-deficient hepatocytes (26). These authors suggested that Western diets, frequently poor in thiamine and high in sugars, could result in increased levels of endogenous glyoxals that may lead to predisposition to advanced glycation end product-related pathology and neoplasia. 
Perhaps, in the light of more modern knowledge, it is possible to state that high simple carbohydrate malnutrition can cause symptoms of early beriberi. Since beriberi conjures up an unacceptable concept in the mind of many modern physicians it is probable that it would not be considered in differential diagnosis. It is very likely that many of the poorly understood symptomatology seen today that responds to nutrient therapy is caused by a mixture of marginal classic nutritional diseases, including beriberi, pellagra and scurvy. In our experience it is certainly true that symptoms arising from autonomic dysfunction are usually reversible by nutritional therapy. These symptoms are not infrequently associated with mitral valve prolapse, also associated with dysautonomia (27).

Lonsdale and co-workers $(1,28)$ have published evidence of TD in modern clinical practice due to high calorie, simple carbohydrate malnutrition. The symptoms generated by this kind of malnutrition fall into the range of traditionally diagnosed functional disease. Generally speaking, the deficiency is marginal and this might be defined as a thiamine pyrophosphate effect (TPPE) of between 15 and $25 \%$ in measuring erythrocyte transketolase (17). A patient receiving intravenous hyperalimentation contracted Wernicke's encephalopathy. Although there is a profuse literature on the association of alcoholism with TD and Wernicke's encephalopathy, the encephalopathy in this patient had occurred in spite of the fact that she had been receiving $25 \mathrm{mg}$ of thiamine in the intravenous solution (29). Although alcohol-associated Wernicke-Korsakov syndrome is the most frequently encountered manifestation of TD in Western culture, it is also seen without alcoholism. Poor nutrition, gastrointestinal disease and AIDS can cause the syndrome. The pathology is restricted to the central nervous system (CNS) (30). TD has been reported in association with parenteral nutrition in 11 neonates (31), suggesting that the lesion is etiologically associated with an inability to oxidize the high calorie input.

Necropsy studies have suggested that TD is underdiagnosed in life because the classical clinical presentations are either uncommon or unrecognized. Marginal TD was found in $31 \%$ and definite deficiency in $17 \%$ of 36 non-demented, community-dwelling patients admitted to an acute geriatric unit (32). There is no doubt that severe TD is lethal but marginal deficiency can give rise to symptoms that are commonly mistaken for functional disease $(1,28)$. A very early experiment in human subjects showed that marginal deficiency produced a multitude of symptoms that might, under ordinary clinical conditions, be regarded as functional in nature. The symptoms disappeared quickly when a thiamine sufficient diet was restored (33). Animal studies have shown that rats develop aggressive reflex behavior when marginal thiamine deficiency is induced experimentally $(34,35)$. A live mouse, introduced to the cage where a thiamine deficient rat was housed, would be killed, but thereafter ignored and not consumed. Persistent erection suggested increased autonomic activity. It is possible that this might be a clue to poorly understood aggressive human behavior.
TD has been reported in refractory heart failure (36) and has occurred in epidemic form in Cuba involving 50862 (461.4 per 100000) people. Lesions included peripheral neuropathy, retrobulbar optic neuropathy, sensory and dysautonomic peripheral neuropathy, sensorineural deafness, dysphonia, dysphagia and spastic paraparesis $(37,38)$. TD ataxia (39), post-gastrectomy polyneuropathy (40) and reversible autonomic neuropathy involving bladder dysfunction (41) have been described.

\section{Pathophysiology of Thiamine Deficiency}

The pathophysiology is complex and only partly understood. A conference in 1982 was published that reviewed the existing knowledge of the vitamin at that time (42). In pyrithiamineinduced thiamine deficiency in animal studies, the net levels of phosphocreatine in the lateral vestibular nucleus and other nuclei of brain were increased selectively. The author suggested that this was possibly due to under utilization (43). Alteration was found in the cerebellar high affinity uptake system for serotonin, aspartate and glutamate in rats with chronic or acute TD (44). It was hypothesized that this was related to deterioration of many membranous systems. Gibson et al. (45) concluded that several lines of reasoning implicate the cholinergic system in the pathophysiology of TD.

Experiments in DBA/J2 mice supported this hypothesis (46). In spontaneously remitting audiogenic seizures in neonates of this inbred strain, known to be cholinergically generated, the seizures were more severe and the spontaneous remission delayed by pretreatment with thiamine tetrahydrofurfuryl disulfide (TTFD). Meador et al. (47) found evidence for a central cholinergic effect of thiamine by studying its action on the cognitive deficits induced by scopolamine in healthy young adults and thiamine treatment resulted in a mildly beneficial effect in some patients with Alzheimer's disease (AD) $(48,49)$.

Levels of free thiamine and its esters, TMP and TPP and activities of the three TDP-metabolizing enzymes, thiamine pyrophosphokinase, thiamine diphosphatase and thiamine triphosphatase, were measured in autopsied cerebral cortex of 18 patients with $\mathrm{AD}$, compared with 20 matched controls (50). In the AD group, mean levels of free thiamine and TMP were normal, whereas levels of TDP were reduced by 18-21\%. Activities of the TDP-metabolizing enzymes were normal. The authors suggested that the TDP decrease could be explained by a cerebral cortical deficiency of ATP in AD, since this is needed for TDP synthesis. This slight decrease in AD brain might explain the modest improvement in cognitive status in some AD patients when treated with thiamine.

\section{Central Sleep Apnea and Thiamine Metabolism}

Three of six siblings presented with sleep apnea between the ages of 18 and 26 months. Twin females first had irregular respiration and episodes of apnea. Twin A succumbed to an apneic episode while asleep and the diagnosis of sleep apnea was made in Twin B at a sleep clinic. She died in an apneic 
episode 3 months later. The male sibling died in a similar fashion. A fourth, unrelated child was reported with sleep apnea at the age of 7 weeks and died at 31 months. Lesions were confined to the respiratory centers of the lower brain stem (51). The relationship with thiamine metabolism was indicated by the fact that the fourth child, and family members, reported in this publication tested positive for a TTP inhibitor substance found in their urine (52). The detection of this inhibitor substance, though never identified, was thought to be a specific marker for the diagnosis of Leigh's disease (subacute necrotizing encephalomyelopathy) (53).

Cooper had used the detection of this substance as a diagnostic test for Leigh's disease. We had, however, come to the conclusion that the test was important in a variety of patients with neurological symptoms whose urines were sent to Cooper's laboratory (D. Lonsdale, unpublished data). This we had found to be a potentially important indication for thiamine administration since many of these patients, who did not have Leigh's disease, responded clinically to thiamine supplementation. The test was performed exclusively in Cooper's laboratory and has never been used, to our knowledge, since Cooper abandoned this research years ago. We can only assume that it demonstrated, in some important way, the activity of TTP in the CNS. To date, there is no other way of detecting TTP deficiency in the laboratory.

\section{Sudden Infant Death}

Familial sleep apnea (51) and the association with the TTP inhibitor supports the long considered concept that TD produces a central effect, particularly in the rapidly growing brain of infants and children. The lesions were confined to the brain stem and this is not the autopsy picture of Leigh's disease, the condition most associated with TTP metabolism in brain. It has long been our contention that sudden infant death syndrome (SIDS) is due to brainstem dysfunction related to hypooxidative metabolism (54). There appears to be ample reason for considering thiamine to play an active role in maintaining the physiology of brainstem activity where the complex mechanism that governs automatic respiration is situated. Fehily (55) was the first investigator to find that TD was responsible for sudden death in infants. Her communication indicated that high calorie malnutrition, in the shape of milled rice eaten by the breast feeding mothers of these infants was responsible. This was further explored in more recent years by Read and coworkers in Sydney, Australia (56) and Lonsdale et al. (57-61). Barker and Jordan (62) found that thiamine phosphate ester gradients along the phrenic nerve and recurrent laryngeal nerve in SIDS infants revealed axonal thiamine depletion with net efflux of $\sim 70 \%$ compared with fire death controls and other SIDS nerves. The ester most deficient in the SIDS infants was TTP. Some recent publications discuss the abnormal baroreflex mechanisms (63) and the effect of hypoxia (64) in central hypoventilation syndrome, but the underlying etiology is described as congenital and no biochemical research was associated with their discussions.

\section{Thiamine Deficiency Related to Other Nutrients}

Although the full extent of nutrient interdependency is far from fully known, there is certainly evidence that thiamine activity is related to other non-caloric nutrients. For example, calcium and magnesium deficiency affects thiamine distribution in rat liver (65) and magnesium has a particularly important role since it is reported to aggravate thiamine deficiency (66). Administration of magnesium, high energy phosphates, piracetam and thiamine affected erythrocyte transketolase activity (67). A physiological concentration of magnesium can be shown to regulate TPP binding to the thiA riboswitch RNA (68).

\section{Thiamine Dependency}

A child with intermittent episodes of cerebellar ataxia (69) proved to be the first case of thiamine dependency in the decarboxylating component of pyruvate dehydrogenase deficiency (70). It is important to emphasize that individual episodes of ataxia occurred in this child in association with some stress factor. This could be a simple viral or bacterial infection, a relatively mild head injury or an inoculation. Even sudden exposure to air conditioning could set off symptoms in this patient. The regular dose of thiamine that he required was $600 \mathrm{mg}$ and this needed to be doubled if a stress factor became implicated. Incidents of this nature that are described here as 'stress factors' are usually found to be important in marginal metabolic situations where cofactor dependency exists. Thiamine responsive pyruvate dehydrogenase deficiency has been reported more recently (71). Infection was an initiating mechanism in intermittent branched chain ketoaciduria (maple syrup urine disease) (72) and this disease has been found in some cases to be responsive to thiamine, the cofactor for the enzyme that decarboxylates the branched chain keto acids (73).

An extraordinary case of thiamine dependency came to my attention. A 6-week-old girl was referred with the possibility of her physical appearance being associated with a chromosomal syndrome. Chromosome analysis was normal but there was laboratory evidence that there was thiamine deficiency from an abnormal transketolase. Thiamine supplementation produced an immediate clinical improvement. As she grew, however, her mother found independently that she had to increase the thiamine dose that eventually rose to as high as $7 \mathrm{~g}$ a day. Although never completely normal, she graduated from high school where she had been a member of the marching band. She died at the age of 27 years from toxic shock associated with an infection. The biochemical lesion was never studied (D. Lonsdale, unpublished data).

There seems to be little doubt that there are recurrent illnesses that are interpreted wrongly because of a general failure to recognize an underlying metabolic process. For example, not many physicians might think that recurrent febrile lymphadenopathy would be anything but the symptoms generated by infection. Two patients with this clinical presentation whose health was completely restored by treatment 
with thiamine and a multivitamin have been reported (74). Both children exhibited defective thiamine metabolism and the extended requirement of large doses of this vitamin suggested some form of dependency. This suggests that the brain initiating mechanisms of defense in these children were abnormally sensitive to possibly unknown or unrecognized 'stress factors' that could obviously include microorganisms. Since viral infections are seldom elucidated in clinical practice, it has become an acceptable explanation for such illnesses. To make it worse, an antibiotic is often prescribed on the assumption that a bacterial phase may follow what is considered to be a viral infection initially.

\section{Thiamine Transporter Disease}

The SLC19 gene family of solute carriers have been described $(15,16)$. The molecular family of the blood-brain barrier (BBB) choline tranporter may be elucidated in vitro by its interaction with physiologic thiamine levels, and two cationic transporters at the BBB may be responsible for thiamine brain uptake (75). Thiamine responsive megaloblastic anemia (TRMA) syndrome is an autosomal recessive disorder with features that include megaloblastic anemia, mild thrombocytopenia, leucopenia, sensorineural deafness and diabetes mellitus (76). Mutations in the SLC19A2 gene encoding a high-affinity thiamine transporter protein THTR-1 are responsible for the clinical features associated with TRMA syndrome (77).

A mouse model of the TRMA syndrome, lacking functional SLC19A2, has been generated and unexpectedly found to have a male-specific sterility phenotype. Injection of high-dose thiamine was effective in reversing the spermatogenic failure, suggesting that the absence of the thiamine carrier could be overcome by diffusion-mediated transport at supranormal thiamine concentrations (78). These findings suggest an unexpected and critical role for thiamine transport and metabolism in spermatogenesis.

Three TRMA patients have been reported with heart rhythm abnormalities and structural cardiac anomalies (79). The authors suggest that the metabolic defect of TRMA may shed light on the role of thiamine in common cardiac abnormalities, possibly leading to considering routine thiamine supplementation in commonly observed cardiac failure where an etiology is not clear. Thiamine is in free form in the intestinal lumen and in very low concentrations. Absorption takes place primarily in the proximal part of the small intestine by means of a dual mechanism, which is saturable at low physiological concentration and diffusive at higher. The saturable component is missing in patients with TRMA syndrome, producing a general disturbance of thiamine transport (80).

\section{Laboratory Studies}

Platt (20) reported that all his patients with the mild and subacute forms of beriberi had creatinuria. He was unable to determine whether this was a direct result from the thiamine deficiency or whether it was caused by protein calorie deficiency secondary to anorexia that was a constant feature of the disease. In the early days of investigation of the relationship of TD with beriberi, anorexia was used as an important clinical marker of severity of the disease. Return of appetite heralded clinical improvement (Fig. 1).

For this reason, we performed an experiment in rats to try to determine whether creatinuria was due solely to TD (81). Animals that were in a state of partial starvation, consuming a thiamine sufficient diet in an amount equivalent to that

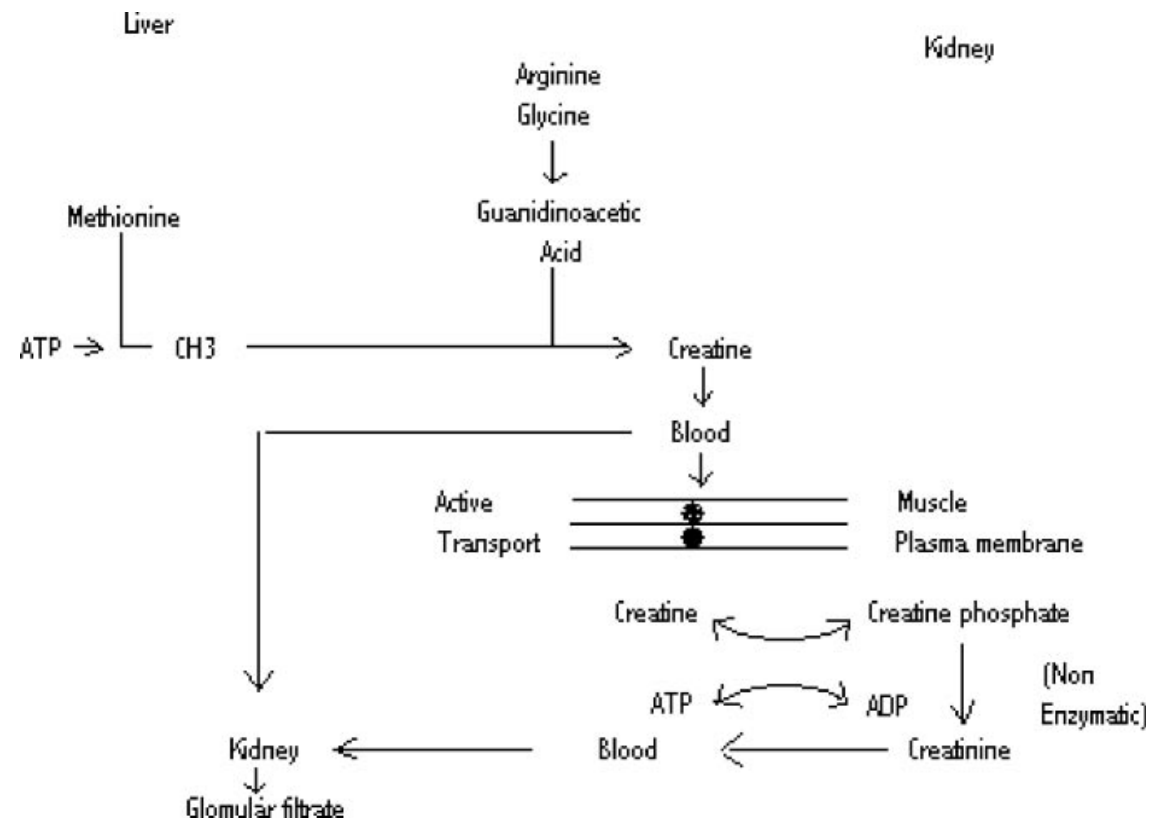

Figure 1. Synthesis, transport and utilization of creatinine and its excretory relationship with creatinine. 
ingested by the anorexic, TD animals, were compared. Increase in creatinuria occurred in both groups as compared with a control group. The increase in creatinuria occurred much earlier in the TD group and was much greater than in the starved animals. We concluded that creatinuria did indeed occur in protein calorie deficiency but that TD had a marked effect on this increase. It suggested cell membrane related phenomena as the underlying cause. It is mindful of the greater degree of creatinuria observed in calorically starved T3 injected rats than in calorically sufficient animals injected with T3 (81).

By examining urine from patients in two $12 \mathrm{~h}$ aliquots, we found that creatine, creatinine and uric acid all followed a pattern of high day/low night concentrations that suggested their response to circadian rhythm (81). Several patients were reported where treatment with thiamine resulted in decreased urinary concentration of creatine and uric acid, while concomitant concentration of creatinine increased (81). This was commensurate with their clinical improvement. This kind of simple test, though by no means specific in indicating TD, was found to indicate biochemical recovery from vitamin therapy, usually accompanied by clinical improvement. Alternating high day and low night concentrations of urinary alanine and pyruvate rose to a peak during an episode of cerebellar ataxia in a child and returned gradually to a normal level as the ataxia slowly and spontaneously improved over an 8 day period. As noted above, this proved to be the first example of thiamine dependency due to compromise of pyruvate decarboxylation $(69,70)$. The mechanism for the day/night alternation of urinary metabolites, though unknown, suggested that circadian rhythm might be involved (81).

The most important laboratory study for TD is measurement of the activity of the enzyme transketolase. Erythrocytes can be used and the test can be made readily available in any commercial laboratory $(1,82-84)$. It has also been reported that transketolase appears to diminish the binding of TPP to the apoenzyme in some patients with Wernicke-Korsakoff syndrome (85). More recently, it has been shown that thiamine metabolism is linked to the pathology in a number of brain diseases and that it is an important indicator of oxidative stress (86). Abnormal transketolase activity has been reported in some autistic children (87). The cornea contains a particularly high transketolase concentration, consistent with the proposal that pentose phosphate pathway activity has a role in the removal of light-generated radicals (88). Abnormal erythrocyte transketolase activity demonstrated that thiamine deficiency was found in a high percentage of HIV-infected patients, not only when in advanced stages but in clinically asymptomatic patients (89).

Neurological disorders in lead intoxicated animals, though not specified by the authors, have been observed in thiamine deficient animals (90). Three-week-old Wistar rats were treated with lead or lead plus thiamine and compared with a thiamine deficient group. Thiamine contents and transketolase activity in the brain were significantly lowered by lead intoxication and thiamine deficiency. In both cases, thresholds of electroshock stimulated seizures were decreased. Thiamine supplementation reversed these signs and decreased the brain lead concentration in the lead treated group (90). Olkowski et al. (91) studied the relative efficacy of thiamine and/or calcium disodium EDTA administration on lead excretion in lead-loaded sheep. They concluded that thiamine enhances elimination of lead from the body and that this feature may be beneficial in chelation therapy. Kim et al. (92) studied the effects of thiamine on the whole body retention of lead in CD-1 mice and concluded that thiamine administration may have therapeutic application for the treatment of lead poisoning. This may be important in treatment of human disease caused by intoxication with $\mathrm{SH}$ reactive metals and suggests that further study is required (87).

\section{Thiaminase}

In 1941, Fujita (93), while engaged in determining the vitamin content of Japanese foodstuffs, found that the thiamine content of some shellfish and crustacea was zero and any added thiamine could not be recovered. He found that the thiamine was being destroyed by an enzyme that he named aneurinase. $\mathrm{He}$ traced the discovery of two enzymes that destroyed thiamine. Thiaminase I (EC 2.5.1.2) splits the pyrimidine from the thiazolium ring at the methylene bridge and attaches a base compound to the pyrimidine ring to form an analog inhibitor of thiamine metabolism. The enzyme is produced by Clostridium thiaminolyticum, an anerobic bacterium found in human small intestine. It is also produced by Bacillus thiaminolyticus, that is aerobic and found in the colon. Bacillus aneurinolyticus is also aerobic, found in the colon and produces thiaminase II (EC3.5.99.2). This enyme functions in the same way as thiaminase I without the attachment of a base compound. Edwin and Gwyneth (94) wrote that thiaminase I was found in the ruminal contents of animals affected by cerebrocortical necrosis. Thiaminases may have a limited part to play in human disease. A case was reported by Murata in 1965 (95). The patient in question had beriberi and potent thiaminase activity was found in the feces. This was then found to be produced by a bacterium in the patient's feces and was termed 'Thiaminase disease'. Seasonal ataxia and impaired consciousness occurs in Nigerians, apparently in epidemic form. A recent study found activity of thiaminase I extracted from the pupae of an African silkworm that is consumed as a source of protein (96). This was the first report of an insect thiaminase and was considered to be the putative cause of the seasonal outbreaks of this disease.

\section{Thiamine Derivatives}

Many thiamine derivatives have been synthesized after the discovery of allithiamine (97) (Figs 2 and 3) Most of the original studies were performed in Japan and the details were published in 1965 (98). Although a series of $S$-acyl derivatives were studied, these investigators found that the disulfides had the best therapeutic effect (99). It was noted that $S$-acyl derivatives are devoid of the preventive effect against trichloroethylene, 
potassium cyanide or lead intoxication that characterized their investigation of thiamine propyl disulfide, one of the early disulfide derivatives. These early investigators ascribed this failure to prevent toxicity to the absence of the S-S bond. The disulfides are easily reduced to thiamine in the presence of cystine or glutathione while the $S$-acyl derivatives require enzymatic reduction in liver or kidney.

The lipophilic derivative known as benfotiamine ( $S$ benzoylthiamine monophosphate, BTMP) has recently received attention and is reported to have clinical benefit in the complications of diabetes (100-103). TTFD is the most modern synthetic disulfide derivative and this has been studied extensively in a variety of clinical conditions (61,87,104-107). What may be of some importance is the disposal of the

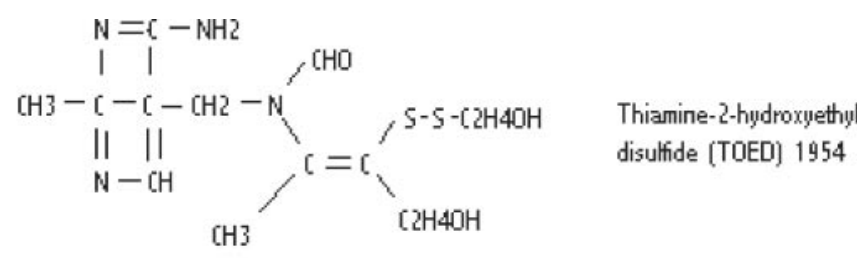

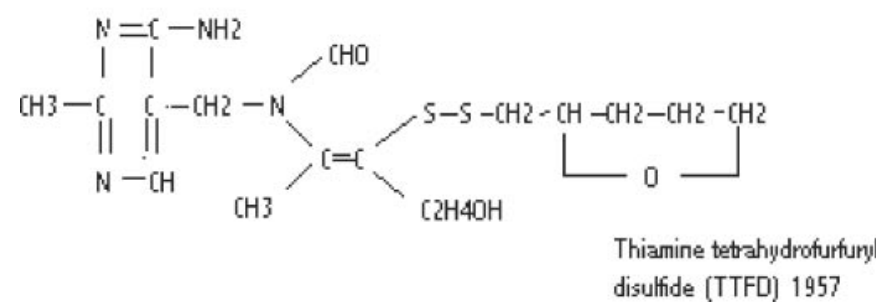

Figure 2. Synthetic allithiamine homologs. prosthetic group that is evolved from any of these derivatives. The mercaptan derived from the disulfide derivatives, that is left outside the cell membrane as the complete molecule is hydrolyzed to deliver thiamine into the cell, has been well studied for its metabolic breakdown (108-112). Its pharmaceutical effect, if any, is unknown.

A poorly known therapeutic use of TTFD may well be in its anti-inflammatory effect. Intraperitoneally administered TTFD and thiamine propyl disulfide (TPD) showed a strong inhibitory effect in carrageenin induced rat paw edema (113). This effect was potentially supported by finding that TTFD reversed the gradual increase in coronary blood flow in the heart-lung preparation of a dog by inhibiting the arachidonic acid cascade-line activation (114).

An open trial with TTFD was performed on 44 patients with polyneuropathy. Thirty-four patients showed improvement of motor function and some restoration of sensory function. Of 18 patients re-examined electrophysiologically 3 months later, 6 showed remarkable improvement. No side effects were observed (115).

Beriberi cardiomyopathy is a well known entity in rice consuming cultures. Three patients were reported with beriberi who presented with different clinical manifestations. The cardiac symptoms responded dramatically to TTFD and there were some improvements in their polyneuropathy measured by somatosensory evoked potentials. These authors stress that the mortality of untreated cardiovascular beriberi is high. In view of the harmless nature of the treatment, they suggested that routine administration of TTFD should be given to all patients in whom heart failure is present without clear evidence of cause (116). In a 12 week open trial, mildly impaired

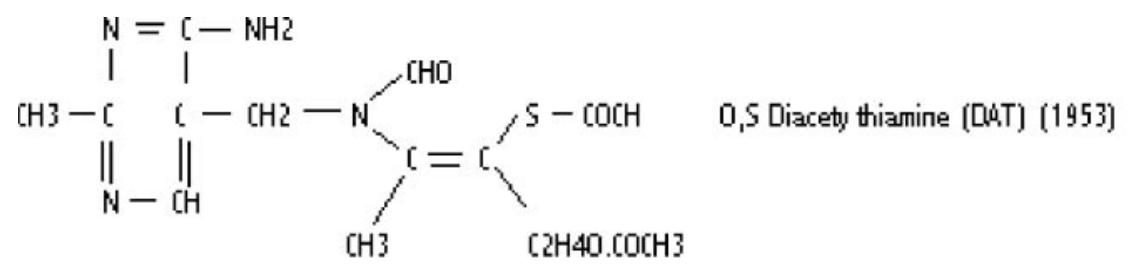

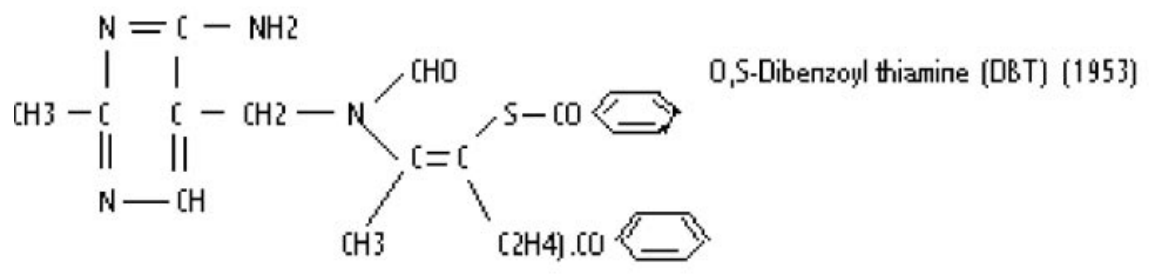

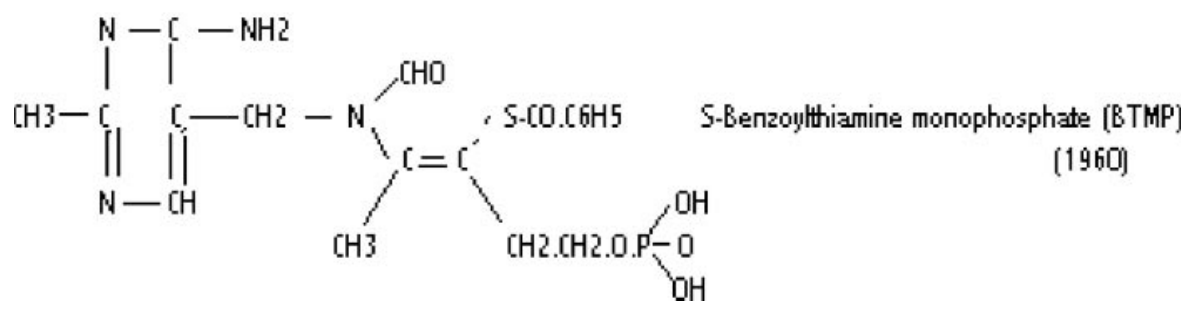

Figure 3. $S$-acyl-thiamine derivatives. 
patients with Alzheimer's disease showed cognitive improvement. No adverse reactions were observed (117).

A test known as the intravenous olfaction test with TPD is a simple procedure widely used in Japan (118). An olfactory stimulus is provided by intravenous injection of TPD and the subject smells $n$-propyl mercaptan, the prosthetic group derived from hydrolysis. This is discharged from blood into the alveoli and expired. The authors recorded electroencephalographic changes during the intravenous olfactory stimulation and found alpha- 2 and beta- 2 waves to be activated over the frontal and temporal regions. The EEGs returned to pre-stimulus levels after disappearance of the olfactory sensation.

It was found that an open thiazole ring thiol form of thiamine (unspecified) released nitric oxide from $S$-nitrosoglutathione (119), a potentially important factor in the therapy of vasculopathy. This might also explain the benefit of thiamine derivatives in the microangiopathy of diabetes (100-103) and certainly deserves further research and clinical trials.

\section{Conclusion and Hypothesis}

The use of thiamine, and its disulfide derivatives in particular, is much neglected in Western medicine. The interest in this vitamin arose in Japan when it was discovered that it had a major part to play in preventing and treating beriberi, a scourge that had affected Eastern cultures for thousands of years. The nutritional diseases have been long associated with poverty and starvation. Starvation, however, represents loss of both calorie yielding and vitamin/mineral containing food and is usually a slow attrition to death. If the calories are maintained without the vitamin/mineral content, also known as empty calories, the disease outcome is very different. Modern Western diet, particularly in children and adolescents, is loaded with simple carbohydrates. In our own clinical experience vitamin deficiency is undoubtedly extremely widespread and thiamine figures very high in this deficiency because of its biochemical association with glucose metabolism $(1,18,24,25,28,54,83,84)$. Because of vitamin fortification of many foods and the relative affluence of our present culture we are not ready to consider that obscure symptoms, particularly those that are generally termed functional, are of dietary origin. They are very frequently the direct result of years of high calorie diet and treatment with large doses of the appropriate vitamin/mineral supplementation is mandatory. Physiological doses have no effect since the enzyme/cofactor bonding appears to be damaged or partially atrophied. Thus, physicians often become disenchanted when they treat a patient with low-dose supplementation and see no benefit. The RDA of thiamine is 1-1.5 mg per day, but only in a biochemically healthy individual, and many physicians are under the impression that this kind of vitamin deficiency, if it occurs at all, is easily and quickly treated. As in the association recorded in this review in the treatment of beriberi, it took very large doses of thiamine for months and there was often only partial recovery and sometimes none at all (20).
It is hypothesized that some crimes and nutritional deficiency are related and that thiamine, because of its association with high carbohydrate intake, including alcohol, may play a significant role. This is certainly not a new concept. Nutritional deficiency and its association with crime has been shown to be an extremely important relationship (120-123). Although the explanation for this has never been attempted, it is suggested that perhaps primitive reflex activity in the limbic system might occur under extreme stress when the supervisory action of the cognitive brain is overwhelmed.

It is worth reminding ourselves that the body is a self-healing 'machine' and all it requires is an appropriate amount of energy to fulfill this amazing role. Vitamins and minerals are to the body as the simple spark plug is to the automobile. The oxidative process requires oxygen, fuel and catalysts and the vitamin/mineral complex is a magnificent effect of millions of years of evolution. We ignore it at our peril.

\section{References}

1. Lonsdale D, Shamberger RJ. Red cell transketolase as an indicator of nutritional deficiency. Am J Clin Nutr 1980;33:205-211.

2. Inouye K, Katsura E. Etiology and pathology of beriberi. In: Shimazono N, Katsura E, (eds). Beriberi and Thiamine. Tokyo: Igaku Shoin Ltd, 1965; 1-28.

3. Jansen BCT, Donath WF. On the isolation of the anti-beriberi vitamin. Proc K Acad Wet Amsterdam 1926;29:1390.

4. Williams RR. Chemistry of thiamine (vitamin B1). JAMA 1938;110: 727-31.

5. Peters RA. The biochemical lesion in vitamin B1 deficiency. Lancet 1936;i:1162-5.

6. Flodin NW. Thiamine (vitamin B1). In: Alan R. (ed). Current Topics in Nutrition and Disease. New York: Liss, Inc., 1988; 103-16.

7. Brin M. Defects of pyruvate and pentose metabolism in relationship to transketolase activity in rats and man and to the startle response in thiamine-deficient rats. In: Wolstenholme GEW, O'Connor M, (eds). Thiamine Deficiency. Boston: Little, Brown and Company, 1967; 87-102.

8. Minz B. Sur la liberation de la vitamin par le tronc isole du nerf pneumogastrique soumis a la excitation electirique. C.R. Soc Biol (Paris) 1938; 127:1251-3.

9. Cooper JR, Pincus JH. The role of thiamine in nervous tissue. Neurochem Res 1979;4:223-39.

10. Iwata H, Yabushita Y, Doi T, Matsuda T. Synthesis of thiamine triphosphate in rat brain in vivo. Neurochem Res 1985;10:779-87.

11. Bettendorff L, Michel-Cahay C, Grandfils C, DeRycker C, Schoffeniels E. Thiamine triphosphate and membrane-associated thiamine phosphatases in the electric organ of Electrophorus electricus. J Neurochem 1987;49:495-502.

12. Bettendorff L, Hennuy B, De Cherek A, Wins P. Chloride permeability of rat brain vesicles correlates with thiamine triphosphate content. Brain Res 1994;652:157-160.

13. Bettendorff L, Kolb HA, Schoffeniels E. Thiamine triphosphate activates anion channels of large unit conductance in neuroblastoma cells. J Membr Biol 1993;136:281-288.

14. Makarchikov AE, Lakaye B, Gulyai IE, Czerniecki J, Coumans B, Wins $\mathrm{P}$, et al. Thiamine triphosphatase and thiamine triphosphatase activities: from bacteria to mammals. Cell Mol Life Sci 2003;60: 1477-88.

15. Ganapathy V, Smith SB, Prasad PD. SLC19: the folate/thiamine transporter family. Pflugers Arch 2004;447:641-6.

16. Nabokina SM, Said HM. Characterization of the $5^{\prime}$-regulating region of the human thiamin transporter SLC19A3: in vitro and in vivo studies. Am J Physiol Gastrointest Liver Physiol 2004;287:G822-9.

17. World Health Organization. Thiamine deficiency and its prevention and control in major emergencies. Report no: WHO/NHD/99.13 Geneva: Department of Nutrition for Health and Development, WHO, 1999. 
18. Fattal-Valegski A, Kesler A, Sela B, Nitzan-Kaluski D, Rotstein M, Mesterman R, et al. Outbreak of life threatening thiamine deficiency in infants in Israel caused by a defective soy based formula. Pediat 2005; 115:es33-8.

19. Lonsdale D. Thiamine metabolism in disease. Crit Rev Lab Sci 1975; 5:289-313.

20. Platt BS. Thiamine deficiency in human beriberi and in Wernicke's encephalopathy. In: Wolstenholme GEW, O'Connor M, (eds). Thiamine Deficiency. Boston: Little, Brown and Company, 1967; 135-43.

21. Inouye K, Katsura E. Clinical signs and metabolism of beriberi patients. In: Shimazono N, Katsura E, (eds). Beriberi and Thiamine. Tokyo: Igaku Shoin Ltd, 1965; 29-63.

22. Lonsdale D. The syndrome of functional dysautonomia. Med Hypoth 1981;7:495-502.

23. Lonsdale D. Asymmetric functional dysautonomia. J Nutr Med 1990; $1: 59-61$.

24. Lonsdale D. Nutritional therapy in children with functional disorders of activity, behavior, attention and learning. Clin Pract Altern Med 2001;2:196-203.

25. Elmadfa I, Majchrzak D, Rust P, Genser D. The thiamine status of adult humans depends on carbohydrate intake. Int J Vitam Nutr Res 2001; 71:217-21.

26. Shangari N, Bruce WR, Poon R, O'Brien PJ. Toxicity of glyoxals-role of oxidative stress, metabolic detoxification and thiamine deficiency. Biochem Soc Trans 2003;31:1390-3.

27. Coghlan HC, Phares P, Cowley M, Copley D, James TN. Dysautonomia in mitral valve prolapse. Am J Med 1979;67:236-44.

28. Lonsdale D. Hypothesis and case reports: possible thiamin deficiency. J Am Coll Nutr 1990;9:13-17.

29. Lonsdale D. Wernicke's encephalopathy and hyperalimentation. JAMA 1978;239:1133.

30. Kril JJ. Neuropathology of thiamine deficiency disorders. Metab Brain Dis 1996;11(1):9-17.

31. Thauvin-Robinet C, Faivre L, Barbier ML, Chevret L, Bourgeois J, Netter JC, et al. Severe lactic acidosis and acute thiamin deficiency: a report of 11 neonates with unsupplemented total parenteral nutrition. J Inherit Metab Dis 2004;27:700-4.

32. O'Keefe ST, Tormey WP, Glasgow R, Lavan JN. Thiamine deficiency in hospitalized elderly patients. Gerontology 1994;40:18-24.

33. Williams RD, Mason HI, Power MH, et al. Induced thiamine (vitamin B1) deficiency in man; relation of depletion of thiamine to development of biochemical defect and of polyneuropathy. Arch Int Med 1943;71:38-53.

34. Onodera K, Tadano T, Sukai K, Kisara K, Ogura Y. Muricide induced by thiamine deficiency in rats. Folia Pharmacol (Japan) 1978; 74:641-8.

35. Onodera K, Kisura K. Persistent erection in thiamine deficient rats. Folia Pharmacol (Japan) 1978;74:649-52.

36. Mendoza CE, Rodriguez F, Rosenberg DG. Reversal of refractory congestive heart failure after thiamine supplementation: report of a case and review of literature. J Cardiovssc Pharmacol Ther 2003; 8: (4)313-6.

37. Roman GC. An epidemic in Cuba of optic neuropathy, sensorineural deafness, peripheral sensory neuropathy and dorsolateral myeloneuropathy. J Neurol Sci 1994;127:11-28.

38. Macias-Matos C, Rodriguez-Ojea A, Chi N, Jimenez S, Zulueta D. Biochemical evidence of thiamine depletion during the Cuban neuropathy epidemic, 1992-1993. Am J Clinc Nutr 1996;64:347-53.

39. Ogunlesi TA. Thiamine deficiency: a cause of childhood ataxia not to be ignored. Ann Trop Paediatr 2004;24:357-60.

40. Kaike H, Iiijima M, Mori K, Hattori N, Ito H, Hirayama M, et al. Postgastrectomy polyneuropathy with thiamine deficiency is identical to beriberi neuropathy. Nutrition 2004;20:961-6.

41. Mazevit D, Vassilev K, Perrigot M. Neuropthy with non-alcoholic thiamine deficiency: two cases of bladder disorders. Ann Readapt Med Phys 2005;48:43-7.

42. Sable HZ, Gubler CJ. (eds). Thiamin: twenty years of progress. Ann NY Acad Sci 1982;378:1-470.

43. McCandless DW. Energy metabolism in the lateral vestibular nucleus in pyrithiamin-induced thiamin deficiency. In: Sable HZ, Gubler CJ, (eds). Thiamin: Twenty Years of Progress. Ann NY Acad Sci 1982;378: 355-64.

44. Plaitakis A, Hwang EC, Van Woert MH, Szilagyi PIA, Beri S. Effect of thiamin deficiency on brain neurotransmitter systems. In: Sable HZ,
Gubler CJ, (eds). Thiamin: Twenty Years of Progress. Ann NY Acad Sci 1982;378:367-81.

45. Gibson G, Barclay L, Blass J. The role of the cholinergic system in thiamin deficiency. In: Sable HZ, Gubler CJ, (eds). Thiamin: Twenty Years of Progress. Ann NY Acad Sci 1982;378:382-403.

46. Lonsdale D. Effect of thiamine tetrahydrofurfuryl disulfide on audiogenic seizures in DBA/J2 mice. Dev Pharmacol Ther 1982;4: 28-36.

47. Meador KJ, Nichols ME, Franke P, Durkin MW, Oberzan RL, Moore EE, et al. Evidence for a central cholinergic effect of high-dose thiamine. Ann Neurol 1993;34:724-6.

48. Blass JP, Gleason P, Brush D, DiPonte P, Thaler H. Thiamine and Alzheimer's disease. Arch Neurol 1988;45:833-5.

49. Meador K, Loring D, Nichols M, Zamrini E, Rivner M, Posas H, et al. Preliminary findings of high dose thiamine in dementia of Alzheimer's type. J Geriatr Psychiatry Neurol 1993;6:222-9.

50. Mastrogiacoma F, Bettendorff L, Grisar T, Kish SJ. Brain thiamine, its phosphate esters, and its metabolizing enzymes in Alzheimer's disease. Ann Neurol 1996;39:585-91.

51. Adickes ED, Buehler BA, Sanger WG. Familial lethal sleep apnea. Hum Genet 1986;73:39-43.

52. Cooper JR, Pincus JH, Itokawa Y, Piros K. Experience with phosphoryl transferase inhibition in subacute necrotizing encephalomyelopathy. N Engl J Med 1970;283:793-5.

53. Cooper JR, Itokawa Y, Pincus JH. Thiamine triphosphate deficiency in subacute necrotizing encephalomyelopathy. Science 1969;164:72-3.

54. Lonsdale D. Sudden infant death syndrome requires genetic predisposition, some form of stress and marginal malnutrition. Med Hypoth 2001:57:382-6.

55. Fehily L. Human milk intoxication due to B1 avitaminosis. Brit Med J 1944;2:590-2.

56. Jeffrey HE, McCleary BV, Hensley WJ, Read DJC. Thiamine deficiency - a neglected problem of infants and mothers-possible relationship to sudden infant death syndrome. Aust NZ H Obst Gynaecol 1985;25:198-202.

57. Lonsdale D, Mercer RD. Primary hypoventilation syndrome. Lancet 1972;ii:487 (Letter).

58. Lonsdale D. Treatment of threatened SIDS with megadose thiamine hydrochloride. Pediat Res 1977;11:379 (Abstr).

59. Lonsdale D. Thiamine deficiency and sudden deaths. Lancet 1990; ii:376.

60. Lonsdale D, Nodar RH, Orlowski JP. The effects of thiamine on abnormal brainstem auditory evoked potentials. Cleve Clin Quart 1979; 46:83-8.

61. Lonsdale D, Nodar RH, Orlowski JP. Brainstem dysfunction in infants responsive to thiamine disulfide; preliminary studies in four patients. Clin EEG 1982;13:82-8.

62. Barker JN, Jordan F. Phrenic thiamin and neuropathy in sudden infant deaths. In: Sable HZ, Gubler CJ, (eds). Thiamin: Twenty Years of Progress. Ann NY Acad Sci 1982;378:449-52.

63. van de Borne P. New evidence of baroreflex dysfunction in congenital central hypoventilation syndrome. Clin Sci (Lond) 2005;108:215-6.

64. Macey PM, Woo MA, Macey KE, Keens TG, Saeed MM, Alger JR, et al. Hypoxia reveals posterior thalamic, cerebellar, midbrain and limbic deficits in congenital central hypoventilation syndrome. J Appl Physiol 2005;98:958-69.

65. Kimura M, Itokawa Y. Effects of calcium and magnesium deficiency on thiamine distribution in rat brain and liver. J Neurochem 1977;28: 389-93.

66. Dyckner T, Elk B, Nyhlin H, Wester PO. Aggravation of thiamine deficiency by magnesium depletion. A case report. Acta Scand 1985; 218:129-31.

67. Eisinger J, Bagneres D, Arroyo P, Plantamura A, Ayavou T. Effect of magnesium, high energy phosphates, piracetam and thiamin on erythrocyte transketolase. Magnes Res 1994;7:59-61.

68. Yamauchi T, Miyoshi D, Kubodera T, Nishimura A, Nakai S, Sugimoto N. Roles of $\mathrm{Mg}^{2+}$ in TPP-dependent riboswitch. FEBS Lett 2005;579:2583-8

69. Lonsdale D, Faulkner WR, Price JW, Smeby RR. Intermittent cerebellar ataxia associated with hyperpyruvic acidemia, hyperalaninemia, and hyperalaninuria. Pediatrics 1969;43:1025-34.

70. Blass J. Abnormalities in pyruvate dehydrogenase and neurologic function. Intern J Neurosci 1972;4:65-9. 
71. Narisawa K, Endo H, Miyabayashi S, Tada K. Thiamine responsive pyruvate dehydrogenase deficiency. J Nutr Sci Vitaminol (Tokyo) 1992;Spec No:585-8.

72. Dancis J, Hutzler J, Rokkones T. Intermittent branched-chain ketonuria: variant of maple-syrup-urine disease. N Engl J Med 1967;276:84-9.

73. Elsas LJ, Danner DJ. The role of thiamin in maple syrup urine disease. In: Sable HZ, Gubler CJ, (eds). Thiamin: Twenty Years of Progress. Ann NY Acad Sci 1982;378:404-20.

74. Lonsdale D. Recurrent febrile lymphadenopathy treated with large doses of vitamin B1: report of two cases. Dev Pharmacol Ther 1980;1: 254-64.

75. Lockman PR, McAfee JH, Geldenhuys WJ, Allen DD. Cation transport specificity at the blood-brain barrier. Neurochem Res 2004;29:2245-50.

76. Ozdemir MA, Alcakus M, Kuroglu, Gunes T, Torun YA. TRMA syndrome (thiamine-responsive megaloblastic anemia): a case report and review of the literature. Pediatr Diabetes 2002;3:205-9.

77. Lagarde WH, Underwood LE, Moats-Staats BM, Calikoglu AS. Novel mutation in the SLC19A2 gene in an African-American female with thiamine-responsive megaloblastic anemia syndrome. Am J Med Genet A 2004; 125:299-305.

78. Oishi K, Barchi M, Au AC, Gelb BD, Diaz GA. Male infertility due to germ cell apoptosis in mice lacking the thiamin carrier, Tht1. A new insight into the critical role of thiamin in spermatogenesis. Dev Biol 2004:266:299-309.

79. Lorber A, Gazit AZ, Khoury A, Schwartz Y, Mandel H. Cardiac manifestations in thiamine-responsive megaloblastic anemia syndrome. Peiatr Cardiol 2003;24:476-81.

80. Rindi G, Laforenza U. Thiamine intestinal transport and related issues: recent aspects. Proc Soc Exp Biol Med 2000;224:246-55.

81. Lonsdale D. Biochemical studies in functional dysautonomia. In: Lonsdale D. (ed). A Nutritionist's Guide to the Clinical Use of Vitamin B1.. Tacoma WA: Life Sciences Press, 1987; 78-115.

82. Boni L, Kieckens L, Hendricx A. An evaluation of a modified erythtocyte transketolase assay for assessing thiamine nutritional adequacy. J Nutr Sci Viatminol 1980;26:507-14.

83. Jeyasingham MD, Pratt O, Burns A, Shaw GK, Thomson AD, Marsh A. The activation of red blood cell transketolase in groups of patients especially at risk from thiamin deficiency. Psych Med 1987;117:311-8.

84. Lonsdale D. Red cell transketolase studies in a private practice specializing in nutritional correction. J Am Coll Nutr 1988;7:61-7.

85. Blass JP, Gibson GE. Abnormality of a thiamine-requiring enzyme in patients with Wernicke-Korsakoff syndrome. N Engl J Med 1977;297: 1367-70.

86. Gibson GE, Zhang H. Interactions of oxidative stress with thiamine homeostasis promote neurodegeneration. Neurochem International 2002;40:493-504.

87. Lonsdale D, Shamberger RJ, Audhya T. Treatment of autistic spectrum children with thiamine tetrahydrofurfuryl disulfide: a pilot study. Neuroendocrinol Lett 2002;23:303-8.

88. Schenk G, Duggleby RG, Nixon FF. Properties and functions of the thiamin diphosphate dependent enzyme transketolase. Int J Biochem Cell Biol 1998;30:1297-1318.

89. Muri RM, Von Overbeck J, Furrer J, Ballmer PE. Thiamin deficiency in HIV-positive patients: evaluation by erythrocyte transketolase activity and thiamin pyrophosphate effect. Clin Nutr 1999;18:375-78

90. Cheong JH, Seo DO, Ryu JR, Shin CY, Kim YT, Kim HC, et al. Lead induced thiamine deficiency in the brain decreased the threshold of electroshock seizure in rat. Toxicology 1999;133:105-13.

91. Olkowski AA, Gooneratne SR, Christensen DA. The effects of thiamine and EDTA on biliary and urinary lead excretion in sheep. Toxicol Lett 1991;59:153-9.

92. Kim JS, Hamilton DL, Blakley BR, Rousseaux CG. The effects of thiamin on lead metabolism: whole body retention of lead-203. Toxicol Lett 1991;56:43-52.

93. Fujita A. Thiaminase. In: Nord FF. (ed.). Adv Enzymol. Interscience Publishers 1954;15:389-421.

94. Edwin EE, Gwyneth L. The implication of ruminal thiaminase in cerbrocortical necrosis. Proc Nutr Sci 1971;330:7A.

95. Murata K. Thiaminase. In: Shimazono N, Katsura E, (eds). Beriberi and Thiamine. Tokyo: Igaku Shoin Ltd, 1965; 220-54.

96. Nishimune T, Watanable Y, Okazaki H, Akai H. Thiamin is decomposed due to Anaphe spp. Entomophagy in seasonal ataxia patients in Nigeria. J Nutr 2000;130:1625-8.
97. Fujiwara M, Watanabe H, Katsui K. Allithiamine, a newly found derivative of vitamin B1. J Biochem 1954;41:29-39.

98. Shimazono N, Katsura E. (eds). Beriberi and Thiamine. Tokyo: Igaku Shoin Ltd., 1965; 1-295.

99. Fujiwara M. Absorption, excretion and fate of thiamine and its derivatives in [the] human body. In: Shimazono N, Katsura E, (eds). Thiamine and Beriberi. Tokyo: Igaku Shoin Ltd., 1965; 179-213.

100. Bitsch R, Wolf M, Moller J, Heuzeroth L, Gruneklee D. Bioavailability assessment of the lipophilic benfotiamine as compared to a water-soluble thiamine derivative. Ann Nutr Metab. 1991;35:292-6.

101. Thornalley PJ. Prevention of incipient diabetic nephropathy by high-dose thiamine and benfotiamine. Diabetes 2003;57:2110-20.

102. Beltramo E, Berrone E, Buttiglieri S, Porta M. Thiamine and benfotiamine prevent increased apaptosis in endothelial cells and pericytes cultured in high glucose. Diabetes Metab Res Rev 2004;20: (4)330-6.

103. Haupt E, Ledermann H, Kopcke W. Benfotiamine in the treatment of diabetic polyneuropathy - a three-week randomized, controlled pilot study (BEDIP study). Int J Clin Pharmacol Ther 2005;43:71-77, Erratum in: Int J Clin Pharmacol Ther 2005;43:304.

104. Lonsdale D. Thiamine metabolism in disease. Crit Rev Lab Sci 1975; 5:289-313.

105. Lonsdale D, Kissling CD. Clinical trials with thiamine tetrahydrofurfuryl disulfide (TTFD) in Down's syndrome. J Orthomol Med 1987; $1: 169-75$.

106. Lonsdale D. Thiamine and its fat soluble derivatives as therapeutic agents. Int Clin Rev 1987;7:114-25.

107. Lonsdale D. Thiamine tetrahydrofurfuryl disulfide: a little known therapeutic agent. Med Sci Monit 2004;10:RA199-203.

108. Kikuchi S, Nishikawa K, Suzuoki Z. The metabolism of thiamine tetrahydrofurfuryl disulfide in the rat, rabbit and man. Eur J Pharmacol 1970;9:367-73.

109. Fujita T, Suzuoki Z. Enzymatic studies on the metabolism of the tetrahydrofurfuryl mercaptan moiety of thiamine tetrahydrofurfuryl disulfide. I Microsomal S-transmethylase. J Biochem 1973;74:717-22.

110. Fujita T, Suzuoki Z, Kozuka S. Enzymatic studies on the metabolism of the tetrahydrofurfuryl mercaptan moiety of thiamine tetrahydrofurfuryl disulfide, II Sulfide and sulfoxide oxygenbases in microsomes. J Biochem 1973;74:723-32.

111. Fujita T, Suzuoki Z. Enzymatic studies on the metabolism of the tetrahydrofurfuryl mercaptan moiety of thiamine tetrahydrofurfuryl disulfide. III. Oxidative cleavage of the tetrahydrofuran moiety. J Biochem 1973;74:733-8.

112. Fujita T, Teraoka A, Suzuoki Z. Enzymatic studies on the metabolism of the tetrahydrofurfuryl mercaptan moiety of thiamine tetrahydrofurfuryl disulfide. IV. Induction of microsomal $S$-transmethylase, and sulfide and sulfoxide oxygenases in the drug-treated rat. J Biochem 1973;74: 739-45.

113. Kitzushima Y. Anti-inflammatory effect of thiamine derivatives. Vitamins (Japan) 1967;36:305-10.

114. Matsui K, Nakahara H, Watanabe J, Tamatsu H, Nakayawa M, Nakayawa Y, et al. Inhibition by thiamine tetrahydrofurfuryl disulfide (TTFD) of the arachidonic acid cascade-line activation as evidenced in the heart-lung preparation of the dog. Jpn J Pharmacol 1985;39:375-79.

115. Djoenaidi W, Notermans SL. Thiamine tetrahdydrofurfuryl disulfide in nutritional polyneuropathy. Eur Arch Psychiatry Neurol Sci 1990;239: 218-20.

116. Djoenaidi W, Notermans SL, Dunda G. Beriberi cardiomyopathy. Eur J Clin Nutr 1992;46:227-34.

117. Mimori Y, Katsuoka H, Nakamura S. Thiamine therapy in Alzheimer's disease. 1996;11:89-94.

118. Harada H, Rikimaru F, Mori T, Tanaka M, Shiraishi K, Kato T. Electroencephalographic changes during intravenous olfactory stimulation in humans. Clin Electroencephalogr 2002;33:189-92.

119. Stepuro AL, Piletskaya TP, Stepuro II. Role of thiamine thiol forms in nitric oxide metabolism. Biochemistry (Mosc) 2005;70:339-49.

120. Schauss A. Diet, Crime and Delinquency. Berkeley, CA 94704: Parker House, 1981; 1-108.

121. Gray GE. Crime and diet: is there a relationship? Wld Rev Nutr Diet 1987;49:66-86.

122. Lonsdale D. Criminal behavior and nutrition. J Adv Med 1992 $5: 115-23$

123. Lonsdale D. Crime and violence: a hypothetical explanation of its relationship with high calorie malnutrition. J Adv Med 1994;7:171-80. 


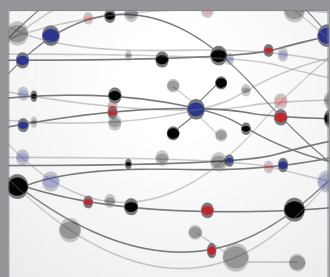

The Scientific World Journal
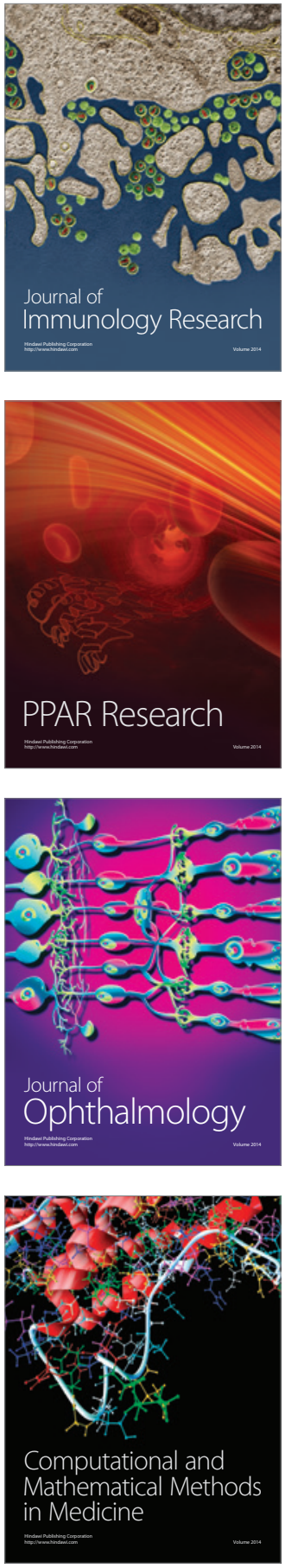

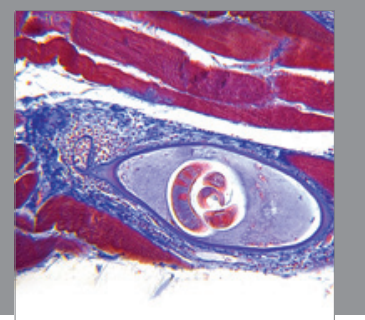

Gastroenterology

Research and Practice
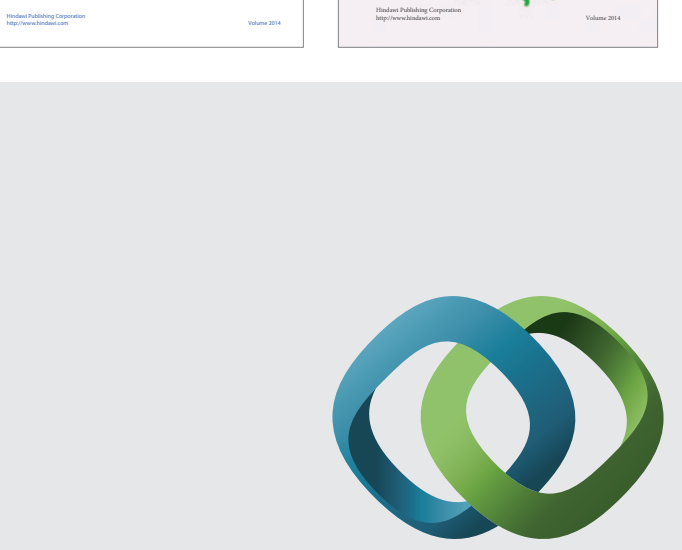

\section{Hindawi}

Submit your manuscripts at

http://www.hindawi.com
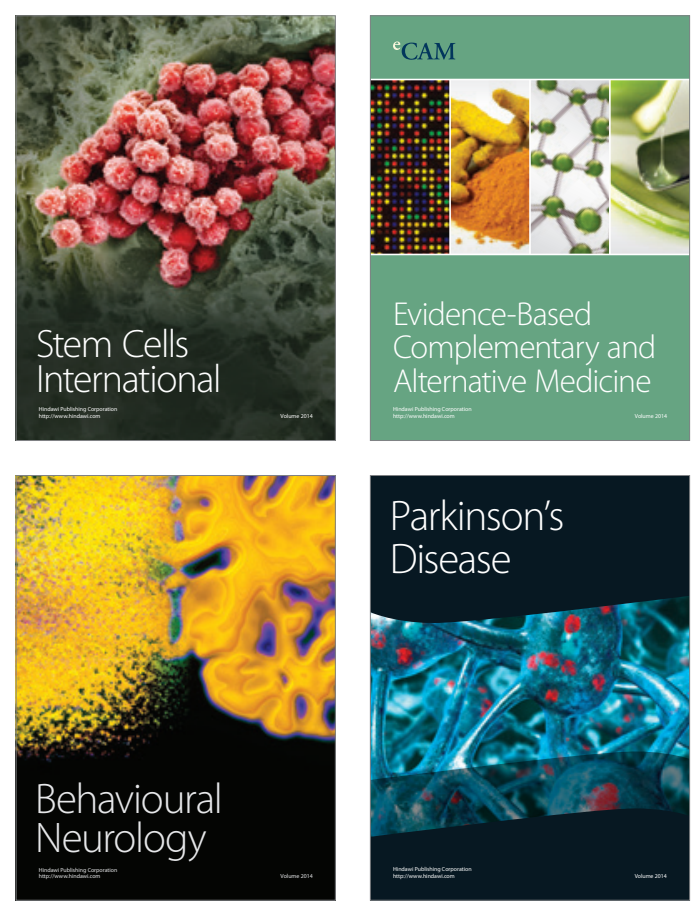

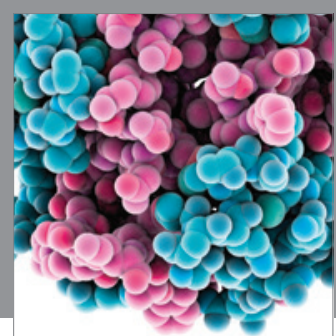

Journal of
Diabetes Research

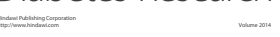

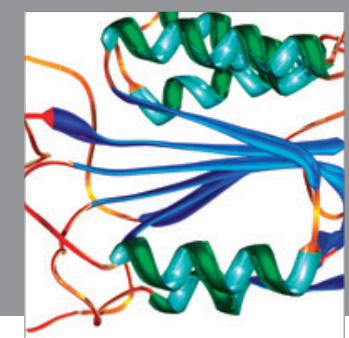

Disease Markers
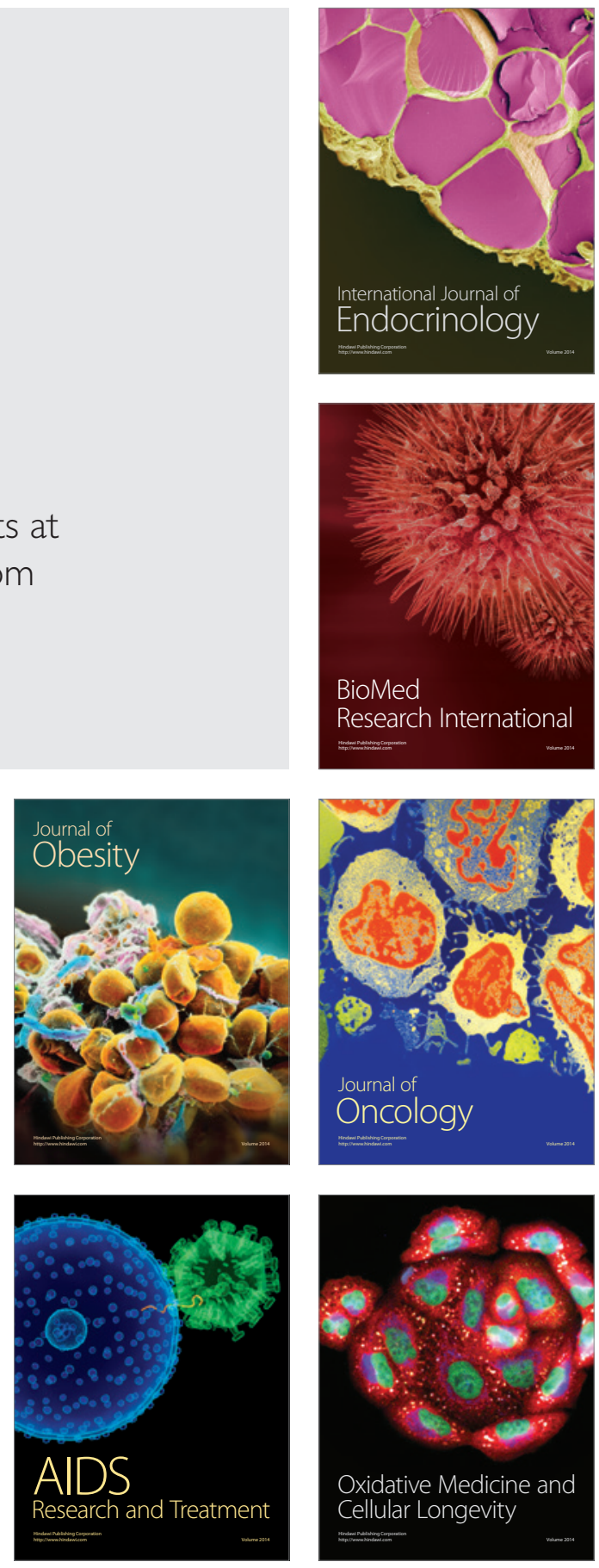